\title{
Multipass beam breakup in energy recovery linacs
}

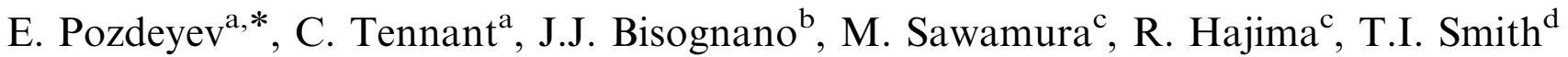 \\ ${ }^{a}$ Thomas Jefferson National Accelerator Facility, 12000 Jefferson Avenue, Newport News, VA 23606, USA \\ ${ }^{\mathrm{b}}$ Synchrotron Radiation Center, Stoughton, WI 53589, USA \\ ${ }^{\mathrm{c} J a p a n}$ Atomic Energy Research Institute Tokai, Ibaraki 319-1195, Japan \\ ${ }^{\mathrm{d}}$ W.W. Hansen Experimental Physics Laboratory Stanford, CA 94305, USA
}

Available online 16 November 2005

\begin{abstract}
This paper is a compilation of several presentations on multipass beam breakup (BBU) in energy recovery linacs (ERLs) given at the 32nd Advanced ICFA Beam Workshop on ERLs. The goal of this paper is to summarize the progress achieved in analytical, numerical, and experimental studies of the instability and outline available and proposed BBU mitigation techniques. In this paper, a simplified theory of multipass BBU in recirculating linacs is presented. Several BBU suppression techniques and their working principles are discussed. The paper presents an overview of available BBU codes. Results of experimental studies of multipass BBU at the Jefferson Laboratory (JLab) FEL Upgrade are described.
\end{abstract}

(C) 2005 Elsevier B.V. All rights reserved.

PACS: 29.27.Bd

Keywords: Energy recovery; Recirculating linacs; Instabilities; Beam breakup; Free electron lasers; Radio frequency

\section{Introduction}

A beam bunch excites dipole higher order modes (HOM) if it passes through a cavity off-axis. The magnetic field of an excited mode deflects the following bunches. The deflection angle produced by the mode translates into a transverse displacement at the cavity after recirculation. The recirculated beam induces an HOM voltage, depending on the magnitude and direction of the beam displacement. Thus, the recirculated beam constitutes a feedback which can become unstable if the beam current reaches the threshold. Because BBU imposes a threat to operations of high-current recirculating machines, better understanding of BBU and development of suppression techniques are tasks of paramount importance.

This paper presents results of analytical, numerical, and experimental studies of multipass BBU conducted by a number of people over an extended period of time. In Section 2, a formula for the BBU threshold for a single dipole HOM with arbitrary polarization in a two-pass

\footnotetext{
${ }^{*}$ Corresponding author. Tel.: + 17572696096 ; fax: + 17572695024 .

E-mail address: pozdeyev@jlab.org (E. Pozdeyev).
}

machine with a general-form, $4 \times 4$ recirculation matrix is derived. Section 2 also contains formulas describing evolution of the HOM voltage above and below the threshold. Section 3 presents an overview of available BBU codes. Section 4 outlines BBU suppressions techniques. The working principles of the mitigation techniques are analyzed on the basis of the theory presented in Section 2. Sections 5 and 6 present results of recent experimental studies of the instability and several BBU suppression techniques at the JLab FEL Upgrade.

\section{Formulas for BBU threshold in a two-pass ERL and HOM voltage behavior above and below the threshold}

\subsection{Single-mode approximation}

Cavity imperfections and asymmetrical features such as couplers violate cylindrical symmetry and split the frequencies of degenerate dipole modes. A typical separation between two orthogonal polarizations of the same dipole mode in a superconducting RF cavity is of the order of several hundred $\mathrm{kHz}$ to several $\mathrm{MHz}$. In addition, imperfections cause HOM frequencies to vary from cavity 
to cavity. The variation of the HOM frequency from cavity to cavity is also of the order of several hundred $\mathrm{kHz}$ to several $\mathrm{MHz}$.

Analytical and simulation results presented elsewhere [1] suggest that a single dipole mode with a quality factor of $10^{5}-10^{6}$ will limit the maximum recirculated current to the order of tens to hundreds of milliamps. Assuming a quality factor of the order of $10^{5}-10^{6}$ and a typical HOM frequency of the order of $2 \mathrm{GHz}$, one concludes that a typical bandwidth of dangerous HOMs is of the order of 1 to $10 \mathrm{kHz}$.

The probability of overlapping of HOM frequencies is small if the number of cavities is significantly smaller than the ratio of the spread of HOM frequencies to the typical bandwidth of the modes. For example, assuming an HOM bandwidth of $10 \mathrm{kHz}$ and a frequency spread of a few $\mathrm{MHz}$, one can consider modes separately if the number of cavities is of the order of a few tens.

\subsection{Two-dimensional formula for the BBU threshold}

Steering and misalignment errors lead to a constant offset of the beam centroid relative to the cavity axis on the first pass. On the second pass, the transverse beam displacement consists of two terms: a constant offset due to steering/misalignment errors and an oscillatory term induced by the HOM voltage on the first pass. The constant offset induces the HOM voltage, which is always limited in amplitude. The oscillatory term, on the contrary, constitutes a feedback that can cause multipass beam breakup and, in theory, an infinite growth of the HOM voltage.

In calculating the threshold of multipass BBU, we treat the voltage induced by the oscillatory term on the second pass as a perturbation and assume that the HOM voltage is a harmonic function of time. A variation of the energy stored in a dipole TM HOM produced by a point-like bunch, $\Delta U$, is given, to the first order in the bunch charge, by [2],

$\Delta U=-q \frac{V_{\mathrm{a}}}{a} \cos (\varphi)(x \cos (\alpha)+y \sin (\alpha))$

where $V_{\mathrm{a}}$ is the accelerating HOM voltage at the beam pipe radius $a$ induced by previous bunches, $\varphi$ is the phase of the point-like bunch with respect to the peak of the HOM electric field, $q$ is the bunch charge, $x$ and $y$ are the horizontal and vertical bunch displacements, and $\alpha$ is the mode polarization angle.

The energy deposited by the bunch in the HOM on the first and second passes can be written as

$$
\begin{aligned}
& \Delta U_{1}=-q \frac{V_{\mathrm{a}}}{a} \cos (\varphi)\left(x_{1} \cos (\alpha)+y_{1} \sin (\alpha)\right), \\
& \Delta U_{2}=-q \frac{V_{\mathrm{a}}}{a} \cos \left(\varphi+\omega T_{\mathrm{r}}\right)\left(x_{2} \cos (\alpha)+y_{2} \sin (\alpha)\right)
\end{aligned}
$$

where $T_{\mathrm{r}}$ is the recirculation time. Here, subscripts 1 and 2 denote the first and second passes respectively. It is assumed in (2)-(3) that the voltage amplitude $V_{\text {a }}$ does not change significantly during recirculation. Exactly at threshold, $V_{\mathrm{a}}$ is constant. Coordinates of the recirculated bunch, $x_{2}$ and $y_{2}$, can be expressed via the bunch coordinates before deflection and the HOM accelerating voltage on the first pass as

$$
\begin{aligned}
x_{2}= & m_{11} x_{1}+m_{12} x_{1}^{\prime}+m_{13} y_{1}+m_{14} y_{1}^{\prime} \\
& -\frac{q V_{\mathrm{a}}}{\omega a p} \sin (\varphi)\left(m_{12} \cos (\alpha)+m_{14} \sin (\alpha)\right) \\
y_{2}= & m_{31} x_{1}+m_{32} x_{1}^{\prime}+m_{33} y_{1}+m_{34} y_{1}^{\prime} \\
& -\frac{q V_{\mathrm{a}}}{\omega a p} \sin (\varphi)\left(m_{32} \cos (\alpha)+m_{34} \sin (\alpha)\right)
\end{aligned}
$$

where $p$ is the beam momentum and $m_{i j}$ are the elements of the recirculation matrix.

The energy balance equation for the HOM stored energy is

$\dot{U}=\dot{U}_{\mathrm{b}}-P_{\mathrm{c}}=\left\langle\Delta U_{1}+\Delta U_{2}\right\rangle \cdot f_{\mathrm{b}}-P_{\mathrm{c}}$.

The averaging is done respectively to the phase of the $\mathrm{HOM}, \varphi$, taken at moments when bunches pass through the cavity on the first pass. Ohmic losses in the cavity can be expressed as [2]

$P_{\mathrm{c}}=\frac{V_{\mathrm{a}}^{2}}{(\omega / c)^{2} a^{2}(R / Q)_{\mathrm{d}} Q}$.

Terms proportional to $\cos (\varphi), \sin (\varphi), \cos \left(\varphi+\omega T_{\mathrm{r}}\right)$, and $\sin \left(\varphi+\omega T_{\mathrm{r}}\right)$ yield zero after averaging if $x_{1}, x_{1}^{\prime}$ and $x_{2}, x_{2}^{\prime}$ in (4)-(5) are steering/misalignment errors. If the HOM frequency is not equal to a harmonic of the bunch repetition rate, terms proportional to $\cos (\varphi) \sin (\varphi)$ also yield zero and the average value of the $\sin ^{2}(\varphi)$ is equal to $\frac{1}{2}$. Thus, Eq. (6) can be rewritten as

$$
\begin{aligned}
\dot{U} & =I_{\mathrm{b}} \frac{q V_{\mathrm{a}}^{2}}{\omega p a^{2}} m^{*}\left\langle\cos \left(\varphi+\omega T_{\mathrm{r}} \sin (\varphi)\right\rangle-\frac{V_{\mathrm{a}}^{2}}{(\omega / c)^{2} a^{2}(R / Q)_{\mathrm{d}} Q}\right. \\
& =-I_{\mathrm{b}} \frac{q V_{\mathrm{a}}^{2}}{\omega p a^{2}} m^{*} \frac{\sin \left(\omega T_{\mathrm{r}}\right)}{2}-\frac{V_{\mathrm{a}}^{2}}{(\omega / c)^{2} a^{2}(R / Q)_{\mathrm{d}} Q}
\end{aligned}
$$

where

$$
m^{*}=m_{12} \cos ^{2}(\alpha)+\left(m_{14}+m_{32}\right) \sin (\alpha) \cos (\alpha)+m_{34} \sin ^{2}(\alpha)
$$

At threshold, $\dot{U}$ is equal to zero. Thus, the threshold current is given by

$I_{\mathrm{b}} \frac{m *}{2 p} \frac{q}{\omega} \sin \left(\omega T_{\mathrm{r}}\right)+\frac{1}{(\omega / c)^{2}(R / Q)_{\mathrm{d}} Q}=0$

which yields the threshold current as

$I_{\mathrm{th}}=-\frac{2 p c}{q(\omega / c)(R / Q)_{\mathrm{d}} Q m^{*} \sin \left(\omega T_{\mathrm{r}}\right)}$.

Precise knowledge of the phase $\varphi$ in (8) is required to find the threshold if the HOM frequency is equal to a harmonic of the bunch repetition rate. Because the presented method does not provide this information, in 
the case of a resonance, one has to calculate the phase, using other methods, or proceed, using computer simulations. Note that the resonance between potentially dangerous HOMs and beam harmonics should be avoided by a proper choice of cavity and beam parameters at the design stage.

For positive values of the product $m^{*} \sin \left(\omega T_{\mathrm{r}}\right)$, Eq. (11) yields a negative threshold that implies absolute beam stability. However, the beam can be unstable at extremely high values of the beam current even if $m^{*} \sin \left(\omega T_{\mathrm{r}}\right)>0$. This discrepancy is caused by the assumption that the voltage induced by the beam on the second pass is a small perturbation to the HOM voltage, which fails at high beam intensities. In one-dimensional cases, a similar dependence of the threshold on $m^{*} \sin \left(\omega T_{\mathrm{r}}\right)$ was predicted analytically and observed in simulations in early works by Krafft et al. [3], Bisognano and Glukcstern [4], Yunn [5], and later by Hoffstaetter and Bazarov [6].

\subsection{Evolution of the HOM voltage below and above the threshold}

Eq. (8) can be rewritten as

$\frac{\mathrm{d} U}{U}=-\mathrm{d} t \frac{\omega}{Q} \frac{I_{\mathrm{th}}-I}{I_{\mathrm{th}}}$.

The solution of the last equation is

$U=U_{0} \exp \left(-t \frac{\omega}{Q} \frac{I_{\mathrm{th}}-I}{I_{\mathrm{th}}}\right)$.

The HOM voltage depends on time as

$V=V_{0} \exp \left(-t \frac{\omega}{2 Q} \frac{I_{\mathrm{th}}-I}{I_{\mathrm{th}}}\right)$

Eqs. (13)-(14) describe evolution of the stored energy and HOM voltage below and above the threshold. Using Eqs. (13)-(14), one can introduce the effective quality factor $Q_{\text {eff }}$ for the beam-HOM system,

$Q_{\text {eff }}=Q \frac{I_{\text {th }}}{I_{\text {th }}-I}$.

At zero current, $Q_{\text {eff }}$ is equal to the HOM quality factor $Q$. Eq. (15) can be also rewritten in terms of the decay time as

$\tau_{\text {eff }}=\tau \frac{I_{\mathrm{th}}}{I_{\mathrm{th}}-I}$.

Above the threshold, the decay time given by (16) changes its sign and becomes the growth time of the instability.

Eqs. (13)-(14) are also valid for negative values of the threshold current given by (11) for $m^{*} \sin \left(\omega T_{\mathrm{r}}\right)>0$ as long as the beam current is not too high and the assumptions made during the derivation of (11) are valid. For negative values of the BBU threshold given by (11), Eqs. (15)-(16) can be rewritten as

$Q_{\mathrm{eff}}=Q \frac{-\left|I_{\mathrm{th}}\right|}{-\left|I_{\mathrm{th}}\right|-I}=Q \frac{\left|I_{\mathrm{th}}\right|}{\left|I_{\mathrm{th}}\right|+I}$ $\tau_{\mathrm{eff}}=\tau \frac{\left|I_{\mathrm{th}}\right|}{\left|I_{\mathrm{th}}\right|+I}$.

The last two equations show that for $m^{*} \sin \left(\omega T_{\mathrm{r}}\right)>0$ the effective quality factor and the decay time of beam oscillations decrease with the beam current.

\section{Overview of BBU codes}

If the number of accelerating cavities is large and frequencies of dipole HOMs overlap, analytical treatment of multipass BBU becomes unfeasible. Parametric dependences given by formulas (11) and (13) can be also invalid. For instance, it was observed in early simulations of multipass BBU at CEBAF that the threshold current for many overlapping modes scales approximately as $Q^{-1 / 2}$ rather than $Q^{-1}$ [7]. In this case, one has to resort to computer simulations to find the threshold of multipass BBU.

\subsection{BBU model used in computer codes}

If the bunch is point-like and the force applied by the HOM can be approximated by a delta-function, the beam behavior is expressed by the following expression [4]:

$$
\begin{aligned}
U_{\mathrm{p}}(n, M)= & T_{n, n-1}^{p p} U_{\mathrm{p}}(n-1, M)+T_{n, n-1}^{p p} G I Z_{n-1} \\
& \times \sum_{r=1}^{n_{\mathrm{p}}} \sum_{k=1}^{M+(p-r) M_{0}-1} U_{\mathrm{r}}\left(n-1, M+(p-r) M_{0}\right. \\
& +k) s_{k}\left(\omega_{n} \tau\right)
\end{aligned}
$$

where $U_{\mathrm{p}}(n, M)$ represents the displacement and momentum of the $M$ th bunch on the $p$ th traversal in the $n$th cavity, $Z_{n}$ is the HOM impedance, $M_{0}$ is the number of bunches in one recirculation, $\omega_{n}$ is the HOM angular frequency, $\tau$ is the elapsed time after HOM is induced, $n_{\mathrm{p}}$ is the number of passes, $n_{0}$ is the cavity number, and $I$ is the average current. $s_{k}\left(\omega_{n} \tau\right)$ in (19) is given by the equation

$s_{k}\left(\omega_{n} \tau\right)=\mathrm{e}^{-k \omega_{n} \tau / 2 Q} \sin \left(k \omega_{n} \tau\right)$

$T_{n, m}^{p q}$ is the transfer matrix, and $G$ is given by

$G=\left(\begin{array}{ll}0 & 0 \\ 1 & 0\end{array}\right)$.

The first term of (19) represents the beam transport to the $n$th cavity from the previous cavity. The product $I Z_{n-1} U_{\mathrm{r}}$ represents the induced HOM field in the previous cavity. The term of $s_{k}\left(\omega_{n} \tau\right)$ describes the HOM field damping and the phase shift. The second term in (19) represents the kick by the HOM field.

There are two approaches to solving this problem: one is beam tracking, and the other one is solution of an eigenvalue problem. The beam tracking method calculates the beam position as a function of time and searches the threshold current by changing the beam current. Several tracking codes have been developed. They include BBU-R developed at JAERI, TDBBU and the new code (unnamed 
yet) developed at JLab, and bi developed at Cornell University. The eigenvalue method converts the beam transport equation (19) to an eigenvalue equation and solves the eigenvalue problem. The code MATBBU, developed at JLab, is based on the eigenvalue approach.

\section{2. $B B U$ codes}

\section{$T D B B U$}

The code TDBBU [8-10] was originally developed to predict the transverse $\mathrm{BBU}$ instability threshold in recirculating and energy recovery linacs. In the code, beam bunches are propagated through a machine and all HOM voltages are updated based on the position of bunches entering accelerating cavities. The code uses $4 \times 4$ transfer matrices and can simulate effects of $x-y$ betatron coupling and arbitrary HOM polarization.

\section{$B B U-R$}

The code BBU-R [11] developed at JAERI handles the transverse BBU by calculating beam position as a function of time after time steps equal to a half period of the rf cycle. It is assumed in the code that bunches are point-like. The code uses $2 \times 2$ transfer matrices to calculate particle coordinates and treats $x$ and $y$ planes independently. Presently, the code restricts the machine configuration to 2pass recirculation. Figs. 1 and 2 show results of simulations of the beam dynamics in the JAERI ERL-FEL. While the amplitude of beam oscillations is almost constant in the stable case, it is increasing in the unstable case. Fig. 3 shows the simulated BBU threshold current in the proposed 6-GeV JAERI ERL as a function of the HOM frequency spread for a cavity gradient of $15 \mathrm{MV} / \mathrm{m}$. The 6$\mathrm{GeV}$ ERL will consists of 48 cryostats containing $1.3 \mathrm{GHz}$ superconducting cavities, and 47 quadrupole triplets between cryostats. The threshold current is $\sim 30 \mathrm{~mA}$ for no HOM frequency spread, and $150 \mathrm{~mA}$ for an HOM frequency spread of $1 \mathrm{MHz}$.

\section{New code developed at JLab}

The newly developed BBU code [12] at JLab (yet to be named) simulates the multipass, multibunch BBU instability in two dimensions for HOMs oriented at arbitrary

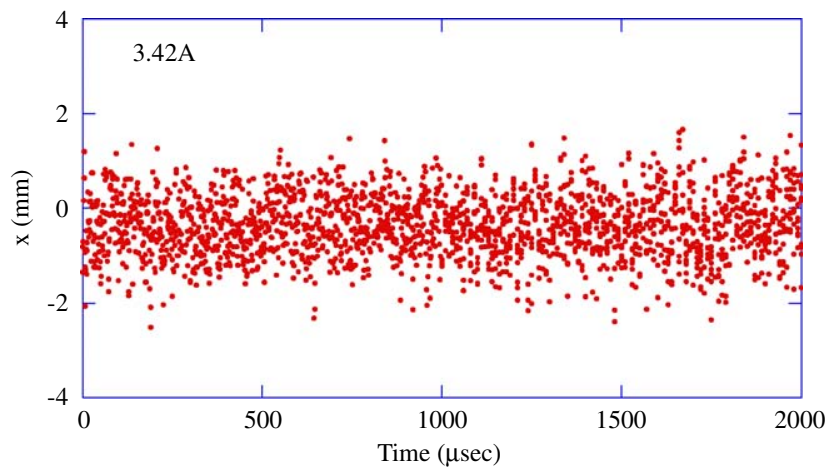

Fig. 1. Results of simulations of the beam dynamics in the JAERI ERLFEL: stable beam. The code BBU-R was used for the simulations.

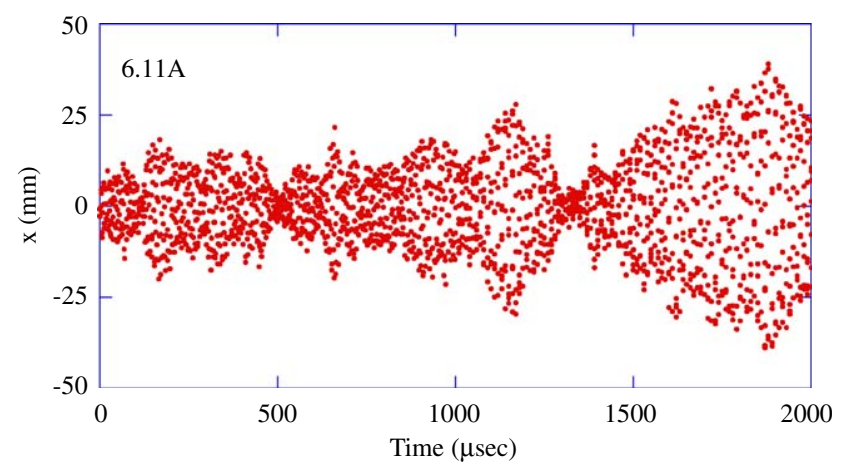

Fig. 2. Results of simulations of the beam dynamics in the JAERI ERLFEL: unstable beam.

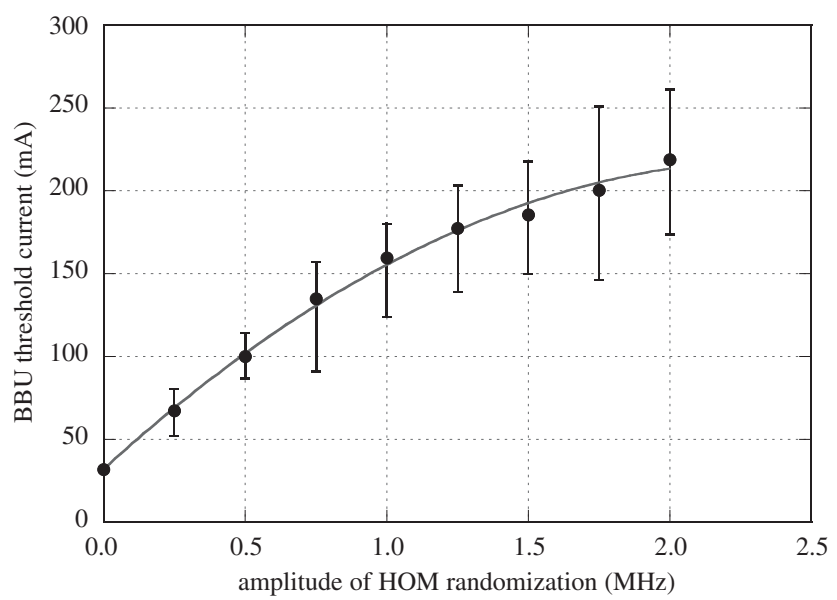

Fig. 3. The threshold current as a function of the HOM frequency spread for the proposed 6-GeV JAERI ERL.

angles and with a full $4 \times 4$ recirculation matrix. Therefore this code has the capability of simulating effects of rotated HOMs and rotated optics on the threshold current. The current version of the code simulates multipass BBU in two pass-accelerators.

bi

The code "bi" [13] was developed at Cornell University. This code can simulate either transverse or longitudinal multipass BBU, as well as transient effects for arbitrary bunch patterns due to interaction with HOMs of the accelerating structures. The code sets up a physical model of the linac in which the bunch train sees a string of cavities. The voltage of HOMs in the cavities is updated in a consistent manner with proper time decays. The code uses $6 \times 6$ transfer matrices and is capable of simulating the beam dynamics with betatron $x-y$ coupling.

\section{$M A T B B U$}

The MATBBU code [14] developed at JLab finds the BBU threshold current solving (19) in the frequency domain. To convert Eq. (19) into the eigenvalue equation the following assumptions are used:

1. There exists a steady state solution of Eq. (19), which can be represented by the product of a steady beam position and an oscillating term. 
Table 1

Main features of the BBU codes

\begin{tabular}{|c|c|c|c|c|c|}
\hline Name & BBU-R & TDBBU & New code & bi & MATBBU \\
\hline Developer & JAERI & JLab & JLab & Cornell Univ. & JLab \\
\hline Transverse/longitudinal & $\mathrm{T}$ & $\mathrm{T}$ & $\mathrm{T}$ & $\mathrm{T} / \mathrm{L}$ & $\mathrm{T}$ \\
\hline Solve & Tracking & Tracking & Tracking & Tracking & Eigenvalue \\
\hline HOM direction & $X, Y$ & $X, Y$ & Arbitrary & Arbitrary & $X, Y$ \\
\hline Dimension & $1 \mathrm{D}$ & $2 \mathrm{D}$ & $1 \mathrm{D} / 2 \mathrm{D}$ & $2 \mathrm{D}$ & $1 \mathrm{D}$ \\
\hline No. of recirculation & 2 & Arbitrary & 2 & Arbitrary & Arbitrary \\
\hline Programming language & $\mathrm{C}$ & Fortran/C & $\mathrm{C}++$ & $\mathrm{C}++$ & Fortran/C \\
\hline
\end{tabular}

2. Number of bunches is large.

3. Summation over the beam positions over the all passes is presented as a separate term.

The converted eigenvalue equation is following:

$$
\begin{aligned}
D_{i}= & I \sum_{p=2}^{n_{p}} \sum_{r<p} \sum_{l=1}^{n_{0}}\left(T_{i, l}^{p r}\right)_{12} \mathrm{e}^{M_{0} \Omega \tau(p-r)} Z_{l} h_{l}(\Omega) D_{l} \\
& +I \sum_{p=1}^{n_{p}} \sum_{l=1}^{i-1}\left(T_{i, l}^{p p}\right)_{12} Z_{l} h_{l}(\Omega) D_{l}
\end{aligned}
$$

where $D_{i}$ represents the beam position summed over the all passes. The $\Omega$ is called the coherent frequency. Since Eq. (21) includes the unknown parameter $\Omega$, the eigenvalues are evaluated at a single frequency. The code sweeps the frequency and calculates the complex eigenvalues of this matrix. The lowest eigenvalue falling on the positive real axis corresponds to the threshold current.

\section{Code comparison}

Table 1 shows the main features of the BBU codes. The beam tracking codes are suitable for the large number of HOMs and a short characteristics time, $(2 Q / \omega)$. Since the growth time of the HOM field is of the order of $\sim 2 Q / \omega$, it takes longer for tracking codes to determine the threshold if high- $Q$ HOMs are simulated.

Calculation time for the eigenvalue algorithm is proportional to $N_{\mathrm{HOM}}^{3.6}$, where $N_{\mathrm{HOM}}$ is the number of HOMs. Thus, simulations can take a long time if the number of cavities is large. Although the simulation time for the eigenvalue method does not depend on the quality factor HOMs for a given frequency, the frequency step must be small enough not to miss HOM resonance peaks. This increases the number of frequency steps if high- $Q$ HOMs are simulated.

\section{Overview of BBU suppression techniques}

BBU suppression techniques can be separated in three groups:

1. Design of cavities with strongly damped HOMs.

2. One and two dimensional optical schemes, including point-to-point focusing and rotation/reflection of the beam displacement.
3. Feedback and other electronic methods increasing damping rates of HOMs.

\subsection{Design of cavities with strong damping of HOMs}

According to (11), the BBU threshold for single HOM in a two-pass machine is inversely proportional to the HOM quality factor. Dipole TM HOMs with a quality factor of the order of $10^{3}-10^{4}$ will limit the maximum recirculated current to hundreds of milliamps to Amperes. Storage rings for light sources and colliders now routinely operate with strongly HOM-damped single-cell cavities. The original CEBAF cavities had a maximum HOM $Q$ of 32000 (a few $10^{4}$ ). Several projects with a goal of developing superconducting multi-cell cavities with a quality factor of dipole HOMs of the order of $10^{3}$ have been started at Brookhaven National Laboratory (BNL), Cornell University, and JLab. Although this method promises to be highly effective in suppressing multipass BBU, a high BBU threshold with such accelerating structures has yet to be experimentally demonstrated.

\subsection{Optical methods}

\section{Point-to-point focusing}

In a two-pass machine with uncoupled recirculation optics, point-to-point focusing from a cavity back to itself will set $m_{12}$ and/or $m_{34}$ to zero yielding an infinite threshold [15]. Such an adjustment of the recirculation optics is routinely used at the JLab FEL Upgrade to increase the threshold of multipass BBU [16]. As shown later in Section 4 , adjusting the phase advance in the recirculation line of the JLab FEL Upgrade, it was possible to significantly increase the threshold and even stabilize some modes. However, point-to-point focusing cannot be arranged for each individual cavity in an extended accelerator with many cavities.

Interchange of $x$ and $y$ planes and $90^{\circ}$ rotation $[15,12]$

A $4 \times 4$ recirculation matrix of the form

$M=\left[\begin{array}{ll}0 & A \\ B & 0\end{array}\right]$

where $A$ and $B$ are $2 \times 2$ block matrices, interchanges the vertical and horizontal betatron planes. If $A$ and $B$ are the 
same, matrix (22) reflects the beam respective to the $x=y$ plane. According to (11), the threshold for the recirculation matrix (22) is inversely proportional to

$m^{*}=\left(m_{14}+m_{32}\right) \sin (\alpha) \cos (\alpha)$.

Thus, if HOMs are bound to the horizontal and vertical planes, an optical insertion with a transfer matrix of the form given by (22) inserted in the recirculation line of a two-pass machine can effectively suppress BBU.

If the $2 \times 2$ block matrix $\mathrm{B}$ in (22) is equal to $-A$, the transformation matrix

$M=\left[\begin{array}{cc}0 & A \\ -A & 0\end{array}\right]$

rotates the beam displacement by $90^{\circ}$. The expression

$m^{*}=m_{12} \cos ^{2}(\alpha)+\left(m_{14}+m_{32}\right) \sin (\alpha) \cos (\alpha)+m_{34} \sin ^{2}(\alpha)$

is always zero for (23), yielding an infinite threshold current regardless the mode polarization angle.

Optic methods based on rotation/reflection of the beam displacement on the second pass can be ineffective if dipole HOMs are degenerate or frequencies of HOMs situated in different cavities overlap. In either case, interference between different HOMs makes assumptions made during derivation of (11) and, as the result, formula (11) itself invalid.

\subsection{Feedback and other electronic methods}

\section{Bunch-by-Bunch, single-pass, broadband feedback}

Transverse bunch-by-bunch feedback is successfully used by high current storage rings such as B-factories for suppression of coupled-bunch instabilities. This type of feedback might be the ultimate cure for multipass BBU in ERLs. A key difference between the two types of instabilities is growth rate, which follows naturally from the fact that $\mathrm{GeV}$-class storage rings might have megavolts of installed RF vs. gigavolts of RF for ERLs of comparable energy. Since ERL cavities will be de- $Q$-ed to the point of substantial overlap among modes of different accelerating units, the coupling impedance simply scales with installed voltage, and growth rates for ERLs (assuming storage-ring-like currents) will be of the order of one circulation time, whereas most storage ring systems deal with 10-100 revolution growth times. For the same transverse error to be corrected, power requirements scale quadratically with growth rate, and quickly the hundreds of watts typical of transverse damping systems can get outlandish. On the other hand, the initial transverse error found in storage rings is generated during the injection process with fast kickers, where many millimeters of error need to be controlled. This is not at all the case with ERLs where the injection process requires no such inflection system, and feed forward or slow feedback systems may allow control of offsets to the micron level. Since power also scales quadratically with high frequency errors, this may bring power requirements under control.
At, say, $1 \mathrm{GHz}$, a large ERL facility would have return times of the order of microseconds, so the delays of $10 \mathrm{~s}$ of nanoseconds possible with modern feedback systems would seem to allow one pass feedback. So, bunch by bunch systems are not precluded. As mentioned before, the growth rates of multipass BBU in ERLs with well damped cavities ( $Q$ of the order of $10^{3}$ ) will be of the order of one revolution time. This requires full correction of errors on each pass; that is, gain of unity. Amplification of noise, with both its effects on beam quality and system power at unity gain becomes the critical issue. Estimates of the required power and an analysis of system stability are needed, and may, in fact, be decisive in determining whether feedback is practical.

Mode-by-mode, narrow-band, beam-based feedback

If the number of cavities of the accelerating structure is small and the frequency of offensive HOMs is well known, a narrow-band, beam-based feedback system can be used to increase the BBU threshold for specific modes. Such a feedback would consist of a stripline pick-up, a receiver that generates the error signal, a narrowband filter, selecting the frequency of a particular sideband produced by an offensive HOM, a phase shifter, a variable attenuator changing the feedback gain, an amplifier, and stripline kicker that applies the correction signal to the beam.

This scheme is capable of suppressing multipass BBU even if the delay time of the feedback loop is larger than the recirculation time and the correction signal is applied to a bunch following the bunch generating the error signal. (It is assumed that the feedback delay time is still significantly shorter than the instability growth time.) A main deficiency of a narrow-band system is that an independent channel is required for each HOM if the frequency separation between modes is comparable to or exceeds $\left(2 \mid T_{\mathrm{d}}-\right.$ $\left.T_{\mathrm{r}} \mid\right)^{-1}$, where $T_{\mathrm{d}}$ is the delay time of the feedback loop and $T_{\mathrm{r}}$ is the recirculation time.

Mode-by-mode, narrow-band, cavity-based feedback

A mode-by-mode, narrow-band, cavity-based feedback was recently tested at the JLab FEL Upgrade. The working principle of this feedback is as follows. A signal from an HOM coupler of a cavity passes through a narrow-band filter centered at the frequency of an offensive HOM. The phase of the signal which passed through the filter is shifted by $\pi$ respective to the input signal by a phase shifter. The gain of loop is adjusted to almost -1 by a combination of a variable attenuator and a low power amplifier. The output of the feedback loop is fed back to the cavity through the same HOM coupler that is used to measure the HOM signal through a high directivity directional coupler.

This feedback was developed as a fast way of mitigating multipass BBU in the JLab FEL Upgrade. The experimental experience with the feedback is described later in this paper.

\section{3-Stub tuners}

Three-stub tuners can be used to suppress multipass BBU. Connected to an HOM port of a cavity and properly tuned, a three-stub tuner reflects the HOM voltage back to 


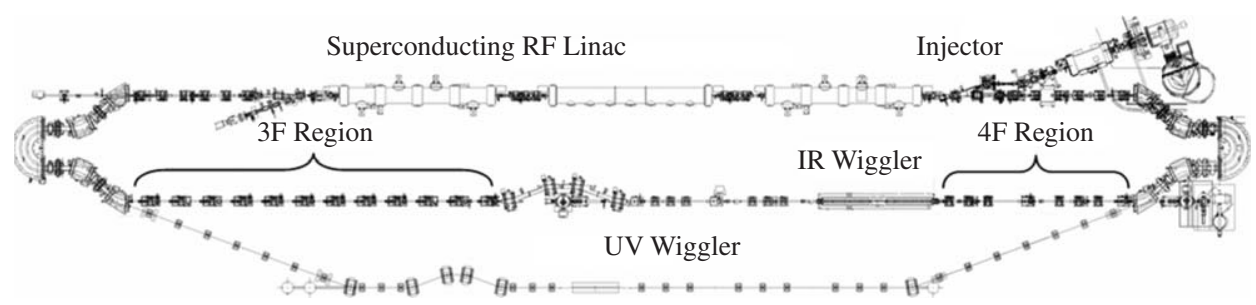

Fig. 4. Layout of the Jefferson Laboratory FEL $10 \mathrm{~kW}$ Upgrade (the UV transport line has not yet been commissioned).

the cavity with a phase advance equal to $\pi$. To be effective, the three-stub tuner has to be connected as close as possible to the HOM port. An experimental experience with a threestub tuner at the JLab FEL Upgrade is described later in this paper.

\section{Experimental characterization of multipass BBU at the JLab FEL upgrade}

The JLab FEL Upgrade is a free electron laser utilizing an energy recovery linac as a driver [17]. The machine consists of a photo-injector, an SRF linac, a recirculation beam line, an FEL, installed in the recirculation line, and a beam dump. A schematic view of the machine is shown in Fig. 4 and the machine parameters are presented in Table 2 .

The SRF linac consists of three cryomodules. The middle cryomodule, frequently referred as Zone 3, was installed in May of 2004 and consists of eight seven-cell cavities. The accelerating gradient of the seven cell cavities is higher than that of five-cell cavities of the side modules (frequently called Zones 2 and 4). The quality factor of dipole HOMs in Zone 3 is also higher than that of HOMs in Zones 2 and 4 by one or two orders of magnitude. That is why dipole HOMs of the middle cryomodule set the BBU threshold in the machine.

Experimental studies of multipass BBU aimed at characterization of the instability were conducted at the JLab FEL Upgrade. The objectives of the studies were:

1. Validation (or refutation) of the model described in Section 2.

2. Validation of the Beam Transfer Function method (BTF) by a comparison of the predicted threshold current with direct measurements.

3. Quantitative comparison of experimental data with analytical results and simulations.

\subsection{Direct observation of multipass $B B U$}

The Zone 3 cryomodule has two HOM ports per each cavity. Cables connected to the HOM ports are loaded on $50 \Omega \mathrm{m}$ resistors as shown in Fig. 5. A small portion of the signal from each HOM port is directed to a Schottky diode through a $20 \mathrm{~dB}$ directional coupler. The time resolution of the diodes is approximately $1 \mu \mathrm{s}$. An output of each diode is
Table 2

JLab FEL Upgrade parameters

$\begin{array}{ll}\text { Energy }(\mathrm{MeV}) & 80-200 \\ \text { Charge per bunch }(\mathrm{pC}) & 135 \\ \text { Bunch rep. rate }(\mathrm{MHz}) & 4-75 \\ \text { Max. average current (mA) } & 10 \\ \text { Laser power }(\mathrm{kW}) & 10\end{array}$

connected to a separate oscilloscope channel. In some cases, the HOM signal after a $20 \mathrm{~dB}$ directional coupler is split further into two. One part of the signal is directed to a Schottky diode to measure the power while the other part is sent directly to an oscilloscope $50 \Omega \mathrm{m}$ input to measure the voltage.

The following procedure was used for observations of the instability. First, the machine was setup for a high current operation in the regime of short (1 ms) pulses. Then, in the $\mathrm{CW}$ regime, the beam current was slowly increased from $0.5 \mathrm{~mA}$ until the beam was lost. The beam was turned off by the machine protection system at a beam current of approximately $2.7 \mathrm{~mA}$ due to high beam losses. These beam loses were accompanied by a growth of the HOM voltage signal from cavity 7 of Zone 3 . Sometimes, it was possible to visually observe a simultaneous growth of the vertical size of the beam spot on the synchrotron light monitor situated in the second $\pi$-bend. Fig. 6 shows the signal and the power of the signal from HOM port 1 of cavity 7 during one of these trips. The signal from the second HOM port of cavity 7 was similar to that from port 1. Calculating FFT of the HOM voltage, we were able to determine the frequency of the signal, which was equal to 2106.01 MHz. This frequency corresponded to the frequency of one of the TM110 HOM in cavity 7. According to preceding measurements of HOM parameters, the quality factor of this mode was one of the highest and was equal to $6 \times 10^{6}$. Thus, using the described technique we were able to determine that the TM110 HOM with the frequency $2106.01 \mathrm{MHz}$ in cavity 7 had the lowest BBU threshold, approximately equal to $2.7 \mathrm{~mA}$. The growth of the vertical size of the beam spot on the synchrotron light monitor situated in the second $\pi$-bend has revealed the vertical polarization of the mode.

Later, the machine was operated in the pulse regime with the beam current increased above $2.7 \mathrm{~mA}$. The beam pulse length was increased from zero until the beam was lost. Fig. 7 shows the logarithm of the HOM power from cavity 


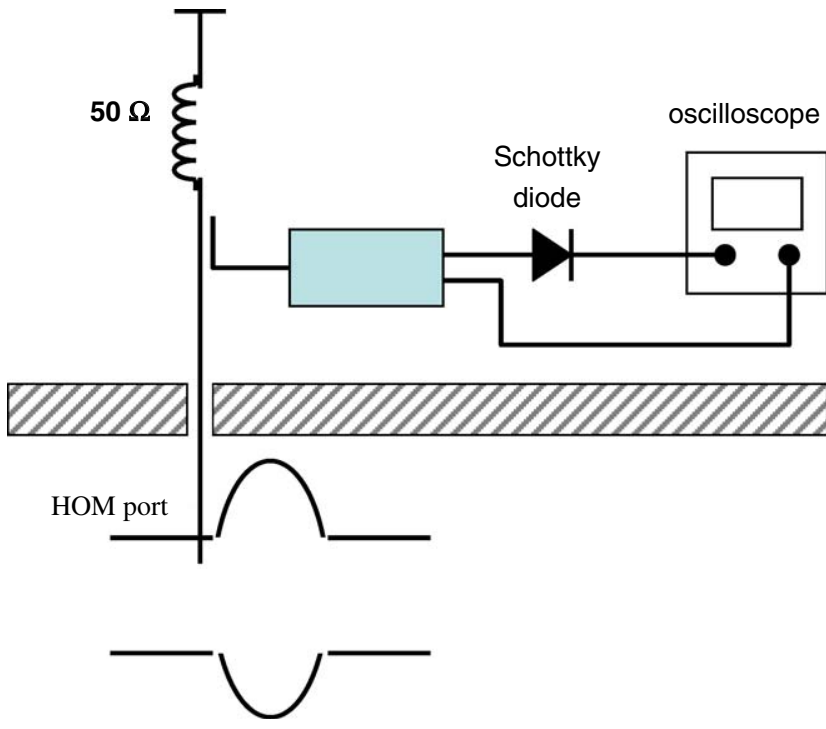

Fig. 5. Experimental setup used for measurements of the HOM voltage and HOM power.

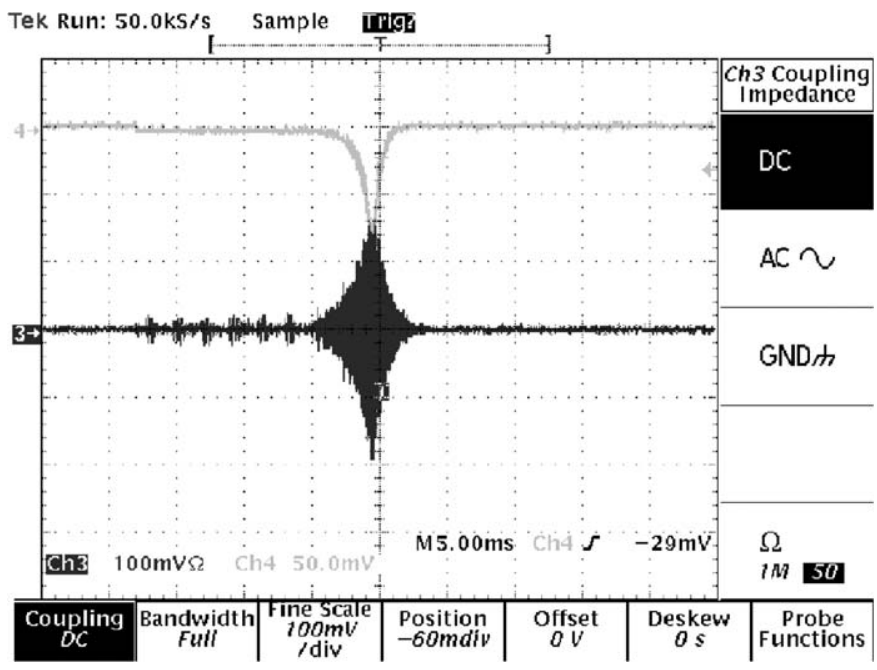

Fig. 6. Voltage (black) and power (grey) of the $2106 \mathrm{MHz}$ HOM in cavity 7 of Zone 3 during multipass BBU. The instability begins at approximately $-18 \mathrm{~ms}$. The HOM voltage grows with the growth rate given by Eq. (16). The beam is lost at the maxiumm of the signal. After the beam is lost, the HOM voltage decays wth the decay time $2 Q / \omega$.

7 for three values of the beam current: $3.5,4.2$, and 5.0 mA. The curves are linear for the upper 90 percent of each curve that reveals an exponential growth of the HOM power and voltage. At the maximum of the curves, the HOM power reached approximately $10 \mathrm{~W}$. Fig. 8 shows the growth rate $(1 / \tau)$ for each curve as a function of the current. According to equation (16), the growth rate of the instability has to be proportional to the difference between the beam current and the threshold current, $I-I_{\mathrm{th}}$. Exactly at the threshold, the growth rate has to be equal to zero. Fitting the growth rate of the HOM power as a function of the current with a straight line and observing where the line crosses the horizontal axis, we obtain the threshold current, $I_{\mathrm{th}}$, equal

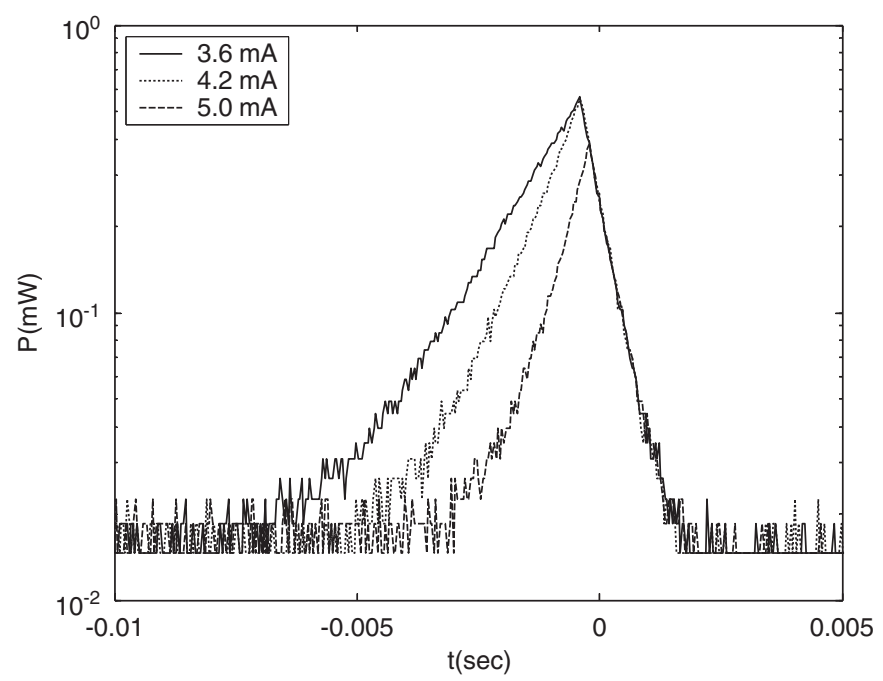

Fig. 7. HOM power of the $2106 \mathrm{MHz} \mathrm{HOM}$ in cavity 7 as a function of time for three different values of the beam current. The measured threshold was $2.7 \mathrm{~mA}$.

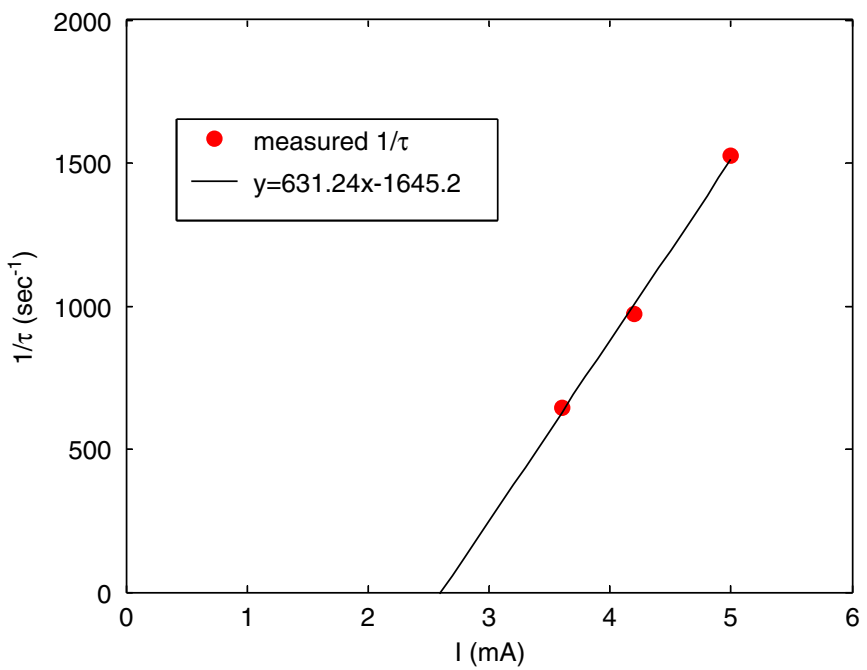

Fig. 8. Growth rate of the HOM power as a function of the beam current. The points correspond to the measured growth rate (see Fig. 7 for details). The line is the linear fit to the experimental data with the equation shown on the graph. This line crosses the horizontal axis at $2.6 \mathrm{~mA}$ that corresponds to the threshold according to Eq. (15). The directly measured threshold was $2.7 \mathrm{~mA}$.

to $2.6 \mathrm{~mA}$, which is very close to the threshold measured directly.

The Schottky diodes connected to cavities 3 and 8 of Zone 3 also detected a growth of the HOM power during the instability. FFT yielded the frequency of the signals: 1706.206 and 1881.481 MHz for cavities 3 and 8, respectively. According to results of earlier HOM measurements, there are high- $Q$ dipole modes in Cavities 3 and 8 with the mentioned frequencies. However, RF simulations predicted $(R / Q)$ for these two modes at least an order of magnitude lower than that of the $2106 \mathrm{MHz}$ mode in cavity 7 . According to analytical estimates and simulations, these 
two modes had to be stable at a beam current of $2.7 \mathrm{~mA}$. This prediction was confirmed for the mode in cavity 3 by BTF measurements described later in this paper. The threshold measured by the BTF method for this mode was approximately $35 \mathrm{~mA}$. This indicates that the mode in cavity 3 was stable during the beam instability. This puzzling result requires additional studies to find the source of the HOM signal from cavities 3 and 8 .

\subsection{Experimental determination of the $B B U$ threshold using the beam transfer function technique}

BTF technique allows one to determine the BBU threshold for individual HOMs, doing measurements below the threshold. In early experiments described in Refs. $[18,19]$, beam oscillations were excited at an injector using a stripline kicker. The cavity response was measured at the frequency of the kicker signal through the cavity probe. The frequency of the signal was swept to measure the transfer function. Dangerous HOMs would appear as resonance peaks in the response signal during the frequency scan. The height of the peaks of dangerous HOMs depended on the beam current. A linear fit of the logarithm of the height of HOM resonance peaks plotted as a function of the logarithm of the beam current was used to determine the threshold.

In the experiments presented in this paper, the beam was excited directly through an HOM port of an accelerating cavity. The response signal was measured from the other HOM port of the same cavity. This technique had a significantly higher signal-to-noise ratio than the method used before and eliminated the need for a kicker and a high-power amplifier. Besides, the quality factor of HOM resonances as a function of the beam current was measured instead of the height of resonance peaks. According to equation (15), the $1 / Q$ has to be a linear function of the beam current and turn to zero exactly at the threshold. Measuring the quality factor instead of the peak height, we have significantly simplified processing of the experimental data. The fact that the beam current in the described below experiments could reach a significant portion of the threshold current also improved accuracy of the BTF measurements.

Fig. 9 shows the resonance curve for the $2106 \mathrm{MHz}$ HOM in cavity 7 measured by a network analyzer for five different beam currents: $0.5,1.0,1.5,2.0$, and $2.5 \mathrm{~mA}$. Fig. 10 shows the measured $1 / Q$ as a function of the beam current. A linear fit to the experimental data yielded a prediction of the threshold at $2.86 \mathrm{~mA}$. This number agrees well with the number measured directly $(2.7 \mathrm{~mA})$ and the number determined from the growth rate of the instability (2.6 mA).

The BTF technique can be used to determine the sign of the product $m^{*} \sin \left(\omega T_{\mathrm{r}}\right)$ in (11). For $m^{*} \sin \left(\omega T_{\mathrm{r}}\right)<0$, the height of the resonance peak growth with the beam current and the quality factor increases. The $1 / Q$ is a linear function of the beam current with a negative slope.

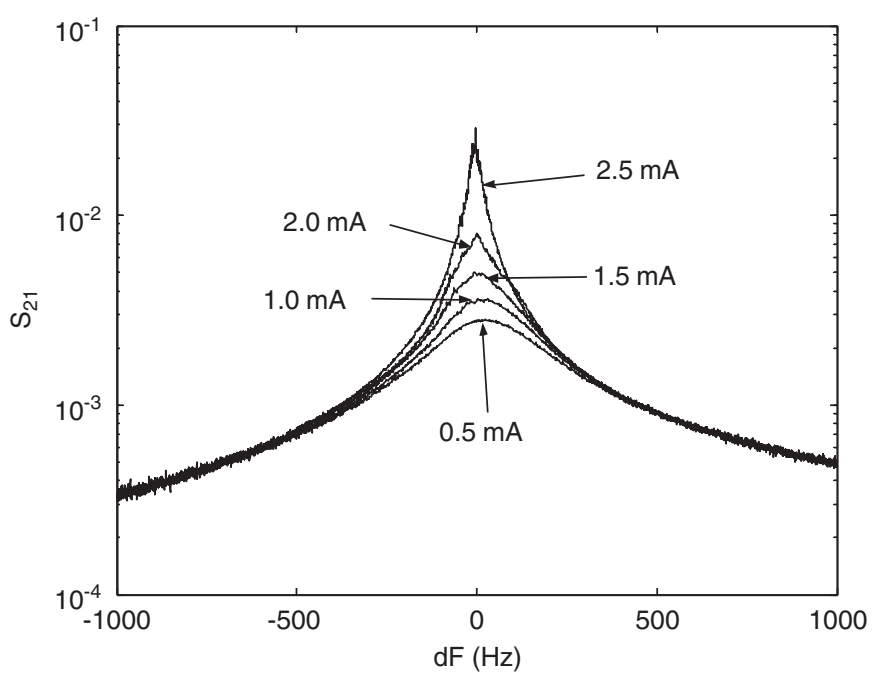

Fig. 9. Resonance curve of the $2106 \mathrm{MHz} \mathrm{HOM}$ in cavity 7 for five different beam currents. The BBU threshold was $2.7 \mathrm{~mA}$.

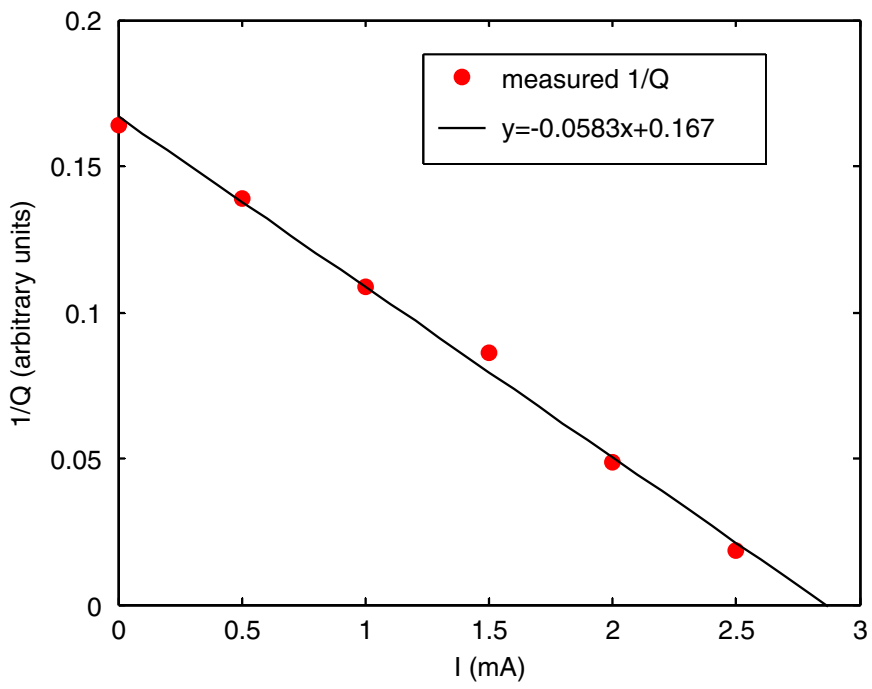

Fig. 10. The quality factor of $2106 \mathrm{MHz}$ mode in cavity 7 as a function of current. The straight line, fitting the data, intersects the horizontal axis at $2.86 \mathrm{~mA}$. The threshold measured directly was $2.7 \mathrm{~mA}$.

According to (17), the slope of the $1 / Q$ changes its sign and becomes positive if $m^{*} \sin \left(\omega T_{\mathrm{r}}\right)>0$. In this case, the height of the resonance peak decreases and the quality factor becomes smaller as the beam current increases. The line $1 / Q(I)$ crosses the horizontal axis at a negative beam current value given by (11). Fig. 11 shows the measured 1/ $Q$ for the $2116.584 \mathrm{MHz}$ TM110 HOM in cavity 7 as a function of the beam current. The line fitting the experimental data has a positive slope and crosses the horizontal axis at $-3.2 \mathrm{~mA}$.

\subsection{Comparison of experimental data to analytical and simulation results}

Multipass BBU was simulated in the optics setup with a measured BBU threshold of $2.7 \mathrm{~mA}$. Three codes were used 


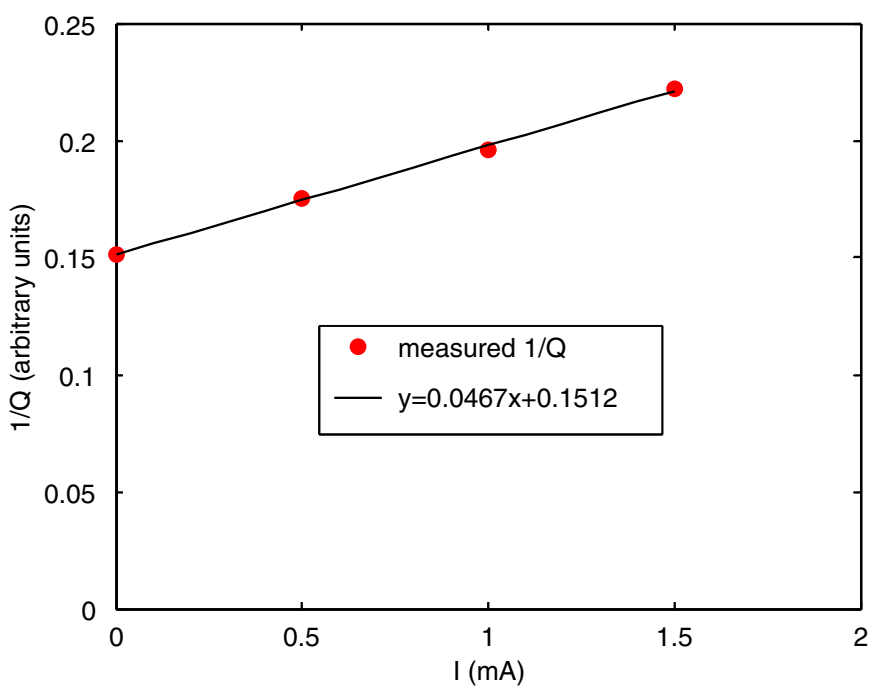

Fig. 11. The quality factor of $2116.584 \mathrm{MHz}$ mode in cavity 7 as a function of current. The straight line, fitting the data, intersect the horizontal axis at $-3.2 \mathrm{~mA}$. The positive slope of the line indicates that $m^{*} \sin \left(\omega T_{\mathrm{r}}\right)$ is positive.

to simulate BBU in the FEL Driver: TDBBU, MATBBU, and the new code developed at Jefferson Lab, briefly described above. The nominal values of the quadrupole strength were used to calculate transfer matrices in the machine. The sixteen highest- $Q$ HOMs of Zone 3, two per each cavity of the cryomodule, were used in simulations. The frequency and quality factor of all the modes were known from earlier RF measurements while the $R / Q$ of the modes was obtained by RF simulations. The $2106 \mathrm{MHz}$ mode in cavity 7 was polarized vertically while polarization of the rest of the modes was set either to zero or $90^{\circ}$. All the three codes yielded the threshold in the range between 2.5 and $2.85 \mathrm{~mA}$, showing a good agreement between the measured data and results of the simulations.

The BBU threshold was also estimated analytically. Eq. (11) was used for each HOM separately. As in the case of simulations, the recirculation matrix was calculated for the nominal set values of the quadrupoles. The $2106 \mathrm{MHz}$ mode in Cavity 7 had the lowest BBU threshold equal to $2.5 \mathrm{~mA}$.

\section{BBU suppression experiments at the JLab FEL upgrade}

In the JLab FEL Upgrade, multipass, multi-pass BBU was observed at a beam current of approximately $2.7 \mathrm{~mA}$. Because BBU imposed an immediate threat to operations of the machine, several BBU suppression techniques were developed and tested. In these experiments, we have studied effectiveness of the following methods: point-topoint focusing, a local reflector consisting of skewquadrupoles, a narrow-band, cavity-based feedback, and a three-stub tuner. Each of these experiments is discussed in detail below.

\subsection{Point-to-point focusing}

Eight quadrupoles following the first $\pi$-bend of the FEL Driver can be used to adjust the betatron phase advance in the recirculation line of the machine. The quadrupoles are used in two sets of four to independently adjust the phase advance in the horizontal and vertical planes. These quadrupole sets are frequently referred to as "phase trombones".

To observe the effect of the phase advance on the BBU threshold the strength of quadrupoles of the vertical phase trombone was changed from its nominal setpoint from -200 to $+300 \mathrm{G}$ in steps of $100 \mathrm{G}$. Fig. 12 shows the measured threshold as a function of the quadrupole strength. The threshold below $5 \mathrm{~mA}$ (the quadrupole strength equal to $-200,-100,0$, and $100 \mathrm{G}$ ) was measured directly by a slow increase of the beam current until the beam was lost. Observing power measured by the Schottky diodes and performing FFT of the unstable signal, we confirmed that the $2106 \mathrm{MHz}$ mode in cavity 7 limited the maximum beam current. For a quadrupole strength of +200 and $+300 \mathrm{G}$, the beam current could not be sufficiently increased to reach the threshold. In this case, the threshold for the $2106 \mathrm{MHz}$ mode in cavity 7 was determined by BTF measurements.

The measured threshold as a function of the quadrupole strength, $G$, exhibits the $(250-\mathrm{G})^{-1}$ dependence. This is explained by the fact that the threshold is inversely proportional to the $m_{34}$. The recirculation optics provides a point-to-point focusing if the quadrupole strength is approximately equal to $+250 \mathrm{G}$. In this case, the phase advance from cavity 7 to cavity 7 is equal $n \pi$, where $n$ is an integer number. For a small variation of the phase advance $\delta$ from $n \pi, m_{34}$ is proportional to the $\delta$ :

$m_{34} \propto \sin (\psi)=\sin (n \pi+\delta) \approx(-1)^{n} \delta$.

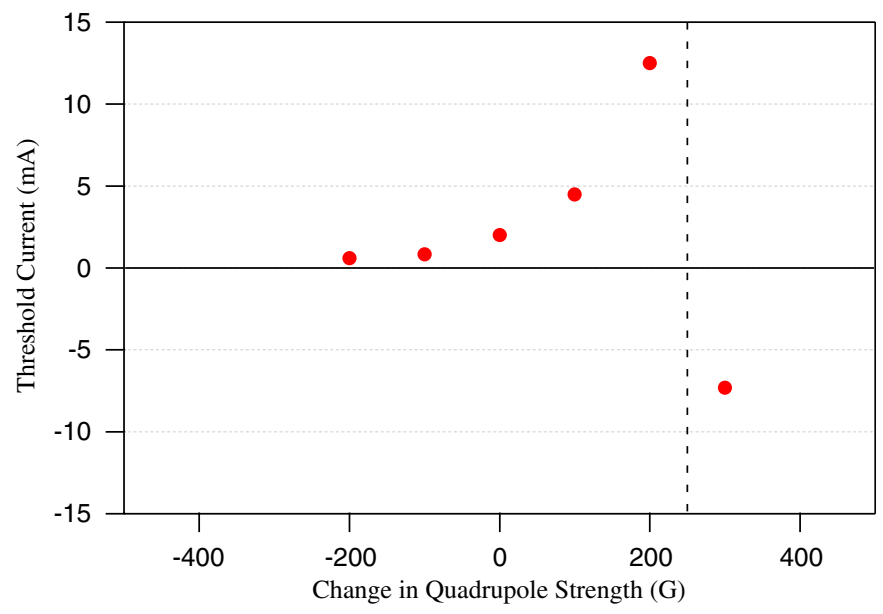

Fig. 12. Measured threshold as a function of the strength of the vertical phase trombone quadrupoles. The threshold for the quadrupole strength equal to +200 and +300 was measured for the $2106 \mathrm{MHz}$ mode in cavity 7 using the BTF technique. Approximately at $G=250$, the $m_{34}$ changes its sign that appears as a negative threshold for $G=300$ measured by BTF. 
The $\delta$, in turn, is proportional to the variation of the quadrupole strength. For the quadrupole strength +300 , the BTF measurements yielded a negative threshold. This indicates that $\mathrm{m}_{34}$ changed its sign and the product $m^{*} \sin \left(\omega T_{\mathrm{r}}\right)$ became positive (for details, see Sections 2.3 and 5.2)

\subsection{Reflection of the beam displacement on the second pass}

In the summer of 2004, a local reflector was noninvasively embedded in the $3 \mathrm{~F}$ region of the FEL Upgrade Driver [20]. The insertion consists of 5 skew-quadrupoles and, when activated, reflects the beam displacement relative to the $y=x$ plane.

To quantify the effect of reflection on BBU, we first measured the threshold current without the reflector, which was $1.8 \mathrm{~mA}$. (The lower threshold was due to minor adjustments in the FEL Driver optics.) The limit was again imposed by the $2106 \mathrm{MHz} \mathrm{HOM}$ in cavity 7. After the reflector was activated, the threshold could not be observed directly. The threshold for the $2016 \mathrm{MHz}$ mode in cavity 7 was determined by BTF measurements. Results of these measurements yielded a new threshold current of $9 \mathrm{~mA}-$ an increase by a factor of 5. Additional BTF measurements performed for two other modes that were a potential threat for the machine operations, the $2116 \mathrm{MHz} \mathrm{HOM}$ in cavity 7 and $2114 \mathrm{MHz}$ mode in cavity 4, indicated that the threshold for both modes was significantly higher than $10 \mathrm{~mA}$.

\subsection{Narrow-band, cavity-based feedback}

The operational principle of the feedback was described in Section 4.3. Experiments aimed at quantifying efficiency of the feedback were conducted as follows. First, the threshold was measured without the feedback. The maximum current was limited to $2.3 \mathrm{~mA}$ by the 2106 HOM in cavity 7 . Then, the beam was turned off and the feedback was activated. The gain and the phase shift of the feedback loop were adjusted to minimize the measured quality factor of the HOM. Adjusting the gain and the phase shift, we were able to reduce the HOM quality factor by a factor of 20 . However, if the suppression factor exceeded 5, the feedback was too sensitive to such disturbances as motion of cables and a warm up of the amplifier and was difficult to operate. After the feedback gain was set to reduce the HOM $Q$ by a factor of 5 , the beam was turned on and the BBU threshold for the 2106 HOM in Cavity 7 was determined by the BTF method. The measured threshold was equal to $10.5 \mathrm{~mA}$, yielding a $\mathrm{BBU}$ suppression factor of 4.5 .

\subsection{Three-stub tuner}

Three-stub tuners were implemented in the way similar to the narrow-band feedback described before. First, the BBU threshold was measured without damping. Than, two stub-tuners were attached to cables connected to HOM ports of Cavity 7. Without the beam, the tuners were adjusted to minimize the quality factor of the $2106 \mathrm{MHz}$ HOM. The quality factor of the HOM was continuously monitored while the position of the stubs was adjusted. A maximum damping factor of 1.5 was achieved. This modest suppression factor is explained by attenuation in the cables from the HOM ports. After the HOM was damped, the beam was turned on and the threshold was re-measured. The threshold with the damping was approximately $50 \%$ higher than the original number measured without damping.

\subsection{Summary of tested suppression techniques}

Table 3 presents a summary of suppression techniques tested at the FEL Driver and their effect on the $2106 \mathrm{MHz}$ mode.

\section{Summary, conclusions, and discussions}

A simplified theory of multipass BBU for a single mode with arbitrary polarization in a two-pass accelerator with a general form $4 \times 4$ recirculation matrix has been presented. The analysis is based on the assumption that the HOM voltage induced on the second pass is a small perturbation to the HOM that deflects the beam on the first pass. Equation (11) accurately describes the BBU threshold for a negative $m^{*} \sin \left(\omega T_{\mathrm{r}}\right)$. For $m^{*} \sin \left(\omega T_{\mathrm{r}}\right)>0$, Eq. (11) yields a negative threshold which implies absolute stability of the beam. However, careful analytical analysis and computer

Table 3

Summary of the effect of BBU suppression techniques applied to the $2106 \mathrm{MHz}$ HOM in the Jefferson Laboratory FEL

\begin{tabular}{llcl}
\hline & \multicolumn{2}{l}{ Threshold current $(\mathrm{mA})$} & \\
\cline { 2 - 4 } Method & Before suppression & After suppression & Suppression factor \\
\hline Damping circuit & 2.3 & 10.5 & 4.5 \\
3-Stub tuner & 2.1 & 3.4 & 1.5 \\
Phase trombone & 2.0 & -7 & 9 \\
Reflector & 1.8 & 9 & 5 \\
\hline
\end{tabular}

The first two methods involve direct damping of the $Q$ whereas the remaining two are examples of beam optical suppression techniques. 
simulations presented in Refs. [3-6] show that the instability can occur at a very high beam intensity (usually at a beam current of the order of $10^{1}-10^{2} \mathrm{~A}$ ) even if $m^{*} \sin \left(\omega T_{\mathrm{r}}\right)>0$. Although the presented theory has a simplified nature, it explains experimentally observed beam behavior in the JLab FEL Upgrade extremely well. Not only the theory accurately predicts the threshold, it also correctly describes evolution of the HOM voltage above and below the threshold, even for HOMs with a positive $m^{*} \sin \left(\omega T_{\mathrm{r}}\right)$ and negative BBU threshold given by (11).

The theoretical analysis presented in this paper is based on the assumption that dipole HOMs are nondegenerate and the number of cavities of the accelerating structure is sufficiently small to neglect interference between HOMs situated in different cavities. To determine the threshold of multipass BBU in machines with a large number of accelerating cavities, one has to resort to computer simulations.

Several computer codes have been developed for simulations of multipass BBU in ERLs and recirculating accelerators. These codes can be divided in two groups based on the solution method: (1) beam tracking and (2) solution of the eigenvalue problem given by Eq. (20). The beam tracking is used by TDBBU, BBU-R, bi, and the new code developed at JLab. MATBBU solves the eigenvalue problem. Although the codes substantially differ from each other in many aspects, they consistently predict similar beam behavior in cross-benchmarks among the codes. Codes TDBBU, MATBBU, and the new code from JLab were used to simulate multipass BBU in the JLab FEL Upgrade. All the three codes predicted the measured BBU threshold with an accuracy of $\pm 10 \%$.

Although a significant progress in BBU simulations has been achieved, additional efforts are required to perform definitive benchmarking of the existing codes to experimental data. These benchmarks will require accurate knowledge of recirculation optics and HOM parameters including HOM polarization. Additional efforts are required to enhance realism of simulations. Such features as monopole modes, beam finite size, beam energy spread, and accurate three-dimensional tracking must be included in future program upgrades.

BBU suppression techniques can be separated in three groups: design of cavities with low- $Q$, low- $R / Q$ HOMs, optical methods, and feedback and other electronic methods that increase damping rates of HOMs.

Cavities with low- $Q$, low- $R / Q$ HOMs, specifically designed for high current operations, are considered as the most promising solution for the BBU problem and currently being developed at Cornell University, BNL, JLab, and, possibly, other laboratories. A threshold current of the order of $0.1-10 \mathrm{~A}$, depending on planned HOM parameters and the number of cavities, is expected.

Optical techniques can be an effective way of suppressing multipass BBU in two pass recirculating accelerators. These techniques minimize the $m^{*}$ given by (9) and include the point-to-point focusing and reflection/rotation of the beam displacement on the second pass. The point-to-point focusing is routinely used at the JLab FEL Upgrade. Although this method proved to be efficient at the JLab Driver, analytical and experimental results presented elsewhere [15] and in this paper suggest that point-to-point focusing can be efficient only for small machines with a small number of cavities. For the reflection or $90^{\circ}$-rotation of the beam displacement to be effective, dipole HOMs have to be nondegenerate and separation between frequencies of HOMs situated in different cavities has to exceed the bandwidth of the modes. These conditions limit applications of the method to compact machines with a small number of cavities or require development of cavities with well controlled HOM frequencies (see for example Ref. [21]).

The beam in large recirculating machines can be susceptible to single-pass, cumulative BBU. The optical methods for multipass BBU damping do not affect cumulative BBU which can limit the ultimate performance of high-current recirculating accelerators after multipass BBU is suppressed by the optical techniques.

A bunch-by-bunch, single-pass, broad-band feedback can be an ultimate solution for the problem of multipass BBU in ERLs. This type of feedback is successfully used by high current storage rings such as B-factories for suppression of coupled-bunch instabilities. Detailed estimates of the required power and analysis of system stability are needed to determine whether broad back feedback is practical for ERLs.

The JLab FEL Upgrade has proven to be a valuable tool for experimental studies of multipass BBU. Several BBU characterization and suppression experiments were successfully conducted at the FEL Driver.

Using Schottky diodes attached to HOM ports of the Zone 3 cavities and performing FFT of the unstable HOM signal, we found that the $2106 \mathrm{MHz}$ TM110 mode in cavity 7 limited the beam current in the machine. An exponential growth of the HOM voltage caused the beam oscillations to grow until the beam was lost. The measured growth rate of the instability agreed well with the growth rate predicted by Eq. (16).

Measuring the quality factor of HOMs as a function of the beam current and using Eq. (15), we were able to accurately determine the BBU threshold for individual modes, conducting the measurements below the threshold. This method, referred to as BTF measurements, was used to quantify suppression techniques in cases when the threshold could not be reached directly. The BTF method was also used to determine a sign of the product $m^{*} \sin \left(\omega T_{\mathrm{r}}\right)$.

The local reflector, consisting of 5 skew-quadrupoles and reflecting the beam displacement respective to the $y=x$ plane, was the most effective way of suppressing the instability. In the nominal setup the reflector pushes the threshold beyond the reachable level. Using the BTF technique, we determined that the reflector increased the threshold of the $2106 \mathrm{MHz}$ mode in cavity 7 by a factor 5 . 
Adjustment of the phase advance in the recirculation line of the FEL Driver is routinely used for BBU suppression. Using the phase trombone, a set of quadrupoles situated in the recirculation line, the threshold can be varied by a factor 4 . Deeper suppression of the instability by the phase trombone is difficult because the product $m^{*} \sin \left(\omega T_{\mathrm{r}}\right)$ depends on the HOM position and frequency. An attempt to stabilize a given mode adjusting the $m^{*}$ will eventually change a sign of $m^{*} \sin \left(\omega T_{\mathrm{r}}\right)$ or increase its magnitude for another mode.

Both the phase trombone and the skew-quad reflector proved to be effective in suppressing multipass BBU in the FEL Driver. However, these methods can produce an undesirable effect on the beam transport tuned for a highpower FEL operation. Electronic methods such as feedback can suppress BBU without affecting the machine optics.

A cavity-based, narrow-band feedback was developed and successfully tested at the JLab FEL Upgrade. This feedback was designed to suppress a single offensive HOM limiting performance of the FEL Driver. Using the feedback, we were able to increase the threshold of the $2106 \mathrm{MHz}$ mode in cavity 7 by a factor of 4.5 .

Three-stub tuners attached to HOM ports of an accelerating cavity can be used to damp cavity HOMs and increase the threshold of multipass BBU. Similar to feedback, three-stub tuners do not affect the beam optics. Using two three-stub tuners, we were able to damp the $2106 \mathrm{MHz}$ mode in cavity 7 by a factor of 1.5 . This low damping factor was due to attenuation produced by long cables connecting HOM ports of the cavity under test with the stub-tuners. A higher damping factor is expected in the case of shorter cables.

\section{Acknowledgements}

The authors gratefully acknowledge the following individuals for their advice, assistance and support during these measurements: Stephen Benson, David Douglas, Curt Hovater, Kevin Jordan, Lia Merminga, Tom Powers, Haipeng Wang and Byung Yunn (Jefferson Lab), Ivan Bazarov, Georg Hofstaetter (Cornell), Stefan Simrock (DESY). This work was supported by The Office of Naval Research the Joint Technology Office, NAVSEA PMS-405, the Air Force Research Laboratory, US Army Night Vision Lab, the Commonwealth of Virginia, and by DOE Contract DE-AC05-84ER40150. The work was also supported by JSPS KAKENHI 15360507.

\section{References}

[1] C. Tennant, Pozdeyev, S. Simrock, A. Sun, H. Wang, JLAB Technical Report TN-04-008, 2004.

[2] H. Padamsee, J. Knobloch, T. Hays, RF Superconductivity for Accelerators, Wiley, New York, 1998.

[3] G.A. Krafft, J.J. Bisognano, S. Laubach, unpublished, 1987.

[4] J.J. Bisognano, R.L. Gluckstern, in: Proceedings of PAC 1987, 1987, p. 1078.

[5] B.C. Yunn, in: Proceedings of PAC 1991, 1991, p. 1785.

[6] G.H. Hoffstaetter, I.V. Bazarov, Phys. Rev. ST Accel. Beams, V7, 054401, 2004.

[7] J.J. Bisognano, private communications.

[8] G.A. Krafft, J.J. Bisognano, in: Proceedings of PAC 1987, 1987, p. 1356.

[9] K.B. Beard, et al., JLAB-TN-02-045, 2002.

[10] K.B. Beard, et al., in: Proceedings of PAC 2003, 2003, p. 332.

[11] M. Sawamura, et al., in: Proceedings of PAC 2003, 2003, p. 3446.

[12] E. Pozdeyev, Phys. Rev. ST Accel. Beams 8 (2005) 054401.

[13] I.V. Bazarov, et al., in Proceedings of EPAC04, 2004, p. 2197, Available at http://lepp.cornell.edu/ ib38/

[14] K.B. Beard, et al., JLAB-TN-02-044, 2002.

[15] R.E. Rand, T.I. Smith, Part. Accel. 11 (1980) 1.

[16] D. Douglas, JLAB Technical Report No. TN-04-017, 2004.

[17] S.V. Benson et al., in: Proceedings of FEL 2004, 2004, Available at http://accelconf.web.cern.ch/accelconf/f04/

[18] N.S. Sereno, Ph.D. Thesis, University of Illinois at UrbanaChampaign, 1994

[19] L. Merminga, et al., in: Proceedings of PAC 2001, 2001, p. 173.

[20] D. Douglas, JLAB Technical Report No. TN-04-016, 2004.

[21] J. Kirchgessner, et al., in: Proceedings of PAC 1989, 1989, p. 479. 